\title{
A CONCEPTUAL DFT STUDY OF THE CHEMICAL REACTIVITY OF MAGNESIUM OCTAETHYLPORPHYRIN (MgOEP) AS PREDICTED BY THE MINNESOTA FAMILY OF DENSITY FUNCTIONALS
}

\author{
Juan Frau ${ }^{\mathrm{a}}$, Francisco Muñoz a and Daniel Glossman-Mitnik ${ }^{\mathrm{a}, \mathrm{b}, *}$ \\ aDepartament de Química, Universitat de les Illes Balears, Palma de Mallorca, Illes Balears 07122, Spain \\ bepartamento de Medio Ambiente y Energía, Centro de Investigación en Materiales Avanzados, Chihuahua, Chih 31136, Mexico
}

Recebido em 05/10/2016; aceito em 21/12/2016; publicado na web em 05/02/2017

\begin{abstract}
The Minnesota family of density functionals has been assessed for the calculation of the molecular structure and electronic properties of a $\mathrm{Mg}(\mathrm{II})$-porphyrin, namely Magnesium Octaethylporphyrin (MgOEP). Several global descriptors arising from Conceptual DFT have been calculated through a $\triangle \mathrm{SCF}$ procedure, and by means of the HOMO and LUMO frontier orbitals. On the basis of the obtained Conceptual DFT indices, a series of descriptors have been devised in order to verify the fulfillment of the "Koopmans' theorem in DFT" procedure. It is shown that the density functionals that verify this approximation with a certain degree of accuracy are only those denoted as range-separated hybrids (RSH), while the usual GGA-hybrids and the local density functionals fail completely.
\end{abstract}

Keywords: Computational Chemistry; Molecular Modeling; Magnesium octaethylporphyrin (MgOEP); Conceptual DFT; Chemical Reactivity Theory

\section{INTRODUCTION}

Porphyrins are large macrocyclic compounds with strong absorbance and fluorescence characteristics. They have been applied in a wide variety of detection approaches due to the sensitivity of those characteristics to their immediate environment. ${ }^{1}$ Porphyrins generally act as carrier molecules for divalent cations such as $\mathrm{Fe}(\mathrm{II})$ in heme, myoglobin and many other heme-containing enzymes like cytochrome and catalase, as well as $\mathrm{Mg}$ (II) in chlorophylls. ${ }^{2}$

Conceptual Density Functional Theory (DFT) or Chemical Reactivity Theory (as it is also known) is a powerful tool for the prediction, analysis and interpretation of the outcome of chemical reactions. ${ }^{3-6}$

Following the pioneering work of Parr and others, ${ }^{3}$ a useful number of concepts have been derived from the analysis of the density of any molecular system through DFT. These concepts that allows a researcher to make qualitative predictions about the chemical reactivity of a given system, can also be quantified and are collectively known as Conceptual DFT Descriptors.

In order to obtain quantitative values of the Conceptual DFT Descriptors, it is necessary to resort to the Kohn-Sham theory through calculations of the molecular density, the energy of the system, and the orbital energies, in particular, those related to the frontier orbitals, that is, the HOMO and LUMO. ${ }^{7-12}$

The usual way to proceed implies as a first step the choice of a model chemistry for the study of the molecular system or chemical reaction of interest. There is a plethora of information in the literature about how to choose this model chemistry and one generally follows the experience of previous researchers and his/her own work.

Although the foundations of DFT have established that a universal density functional must exist, and that all the properties of the system can be obtained through calculations with this functional, in practice one needs to resort to some of the approximate density functionals that have been developed during the last thirty years. Due to the fact that these are approximate functionals (that is, not a universal functional), many of them are good for predicting some properties and others are good

*e-mail: daniel.glossman@ cimav.edu.mx for another properties. Sometimes, you can find density functionals that are excellent for describing the properties of a given molecular system with a particular functional group, but it is necessary to resort to other density functionals for a different functional group that you want to include in the molecular system under study.

When one is dealing with the study of the chemical reactivity, that is, a process that involve the transference of electrons, it is usual to perform calculations not only of the ground state, but also for open systems like the radical cation and radical anion. These systems are often difficult to converge giving trustworthy results, especially if diffuse functions must be included in the basis set. ${ }^{7-12}$ For this reason, it is convenient to have a method that can give all information that one needs directly from the results of the calculation of the ground state of the molecular system under study. In particular, one may want to obtain the ionization potential (I) and electron affinity (A) of the system avoiding the calculation of the energy of the radical anion and cation, because spin-open systems are difficult to converge, in particular, for large molecules. Indeed, the procedure to reach this goal is given by the so-called Koopmans' theorem, ${ }^{9-12}$ that states that within Hartree-Fock (HF) theory, the ionization potential I can be approximated by the negative of the HOMO energy, that is, $\mathrm{I}=-\varepsilon H$. By extension, it is considered that the electron affinity A can be approximated by the negative of the LUMO energy, that is, $\mathrm{A}=-\varepsilon L$. However, the validity of the Koopmans' theorem in DFT is controversial and the problem has been identified with the difference between the fundamental band gap and the HOMO-LUMO gap, that is called the derivative discontinuity. Notwithstanding, it has been mentioned recently ${ }^{13}$ that an exact physical meaning can be assigned to the Kohn-Sham (KS) HOMO using "the KS analog of Koopmans' theorem in Hartree-Fock theory", which states that for the exact theory, the KS HOMO is equal to and opposite of the ionization potential, $\mathrm{\varepsilon H}=$ $-\mathrm{I} .{ }^{14-17}$ Due to the mentioned problem of the discontinuity, a similar Koopmans' theorem that relates the LUMO energy to the electron affinity does not exist. Thus, it has been proposed to circunvect the problem, to consider that the I of the $\mathrm{N}+1$ electron system (the anion) is the same that the $\mathrm{A}$ of $\mathrm{N}$ electron system. ${ }^{13}$ By considering rangeseparated hybrids (RSH) functionals, ${ }^{18-20}$ where the repulsive Coulomb potential is split into a long-range (LR) and short- range (SR) term, 
e.g., via $\mathrm{r}-1=\mathrm{r}-1 \operatorname{erf}(\gamma \mathrm{r})+\mathrm{r}-1 \operatorname{erfc}(\gamma \mathrm{r})$, with $\gamma$ the range-separation parameter, Kronik et al. ${ }^{13}$ showed that with a judicious choice of this last parameter, the validity of the Koopmans' theorem could be enforced. This $\gamma$ tuning technique can be used for the improvement of the description of the properties predicted by these density functionals. This is a consequence of the better fullfilment of the Koopmans' theorem that leads to better agreement of the orbital energies with the I and A. For example, Lima et al. ${ }^{21}$ have recently presented an improved description of the optical properties of carotenoids by tuning some long-range corrected functionals.

This means that the goodness of a given density functional can be estimated by checking how well it follows the "Koopmans' theorem in DFT" that makes it behave closer to the exact density functional, and this will be crucial for a good calculation of the Conceptual DFT descriptors that predict and explain the chemical reactivity of molecular systems. However, the $\gamma$ tuning procedure for the RSH density functionals is system dependent and that implies that different density functionals are going to be used for the calculation of the descriptors for the different molecular systems. Thus, it will be interesting to study other RSH density functionals where the $\gamma$ parameter is fixed by constructions, although other parameters have been fitted to reproduce some molecular properties. In particular, we are going to consider several density functionals that have shown great accuracy across a broad spectrum of databases in chemistry and physics. ${ }^{22}$

The aim of this work is to conduct a comparative study of the performance of the latest Minnesota family of density functionals for the description of the chemical reactivity of a $\mathrm{Mg}$ (II)-porphyrin, namely magnesium octaethylporphyrin (MgOEP), whose molecular structure is shown in Figure 1.

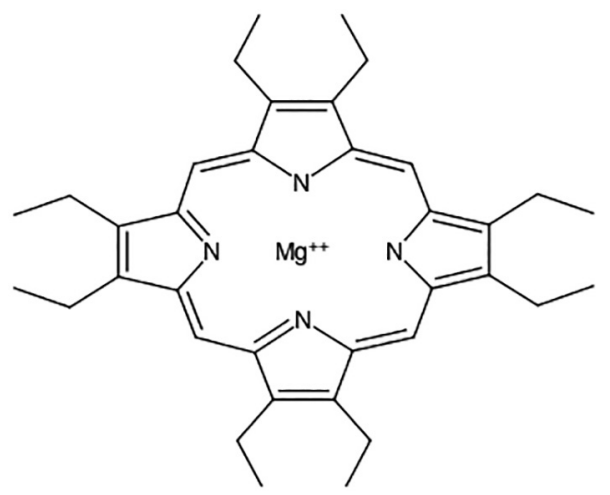

Figure 1. Molecular structure of magnesium octaethylporphyrin (MgOEP)

\section{THEORETICAL BACKGROUND}

Within the conceptual framework of DFT, ${ }^{4,23}$ the chemical potential $\mu$, which measures the escaping tendency of the electron from equilibrium, is defined as:

$$
\mu=\left(\frac{\partial E}{\partial N}\right)_{v(\vec{r})}=-\chi
$$

where $\chi$ is the electronegativity.

The global hardness $\eta$ can be seen as the resistance to charge transfer:

$$
\eta=\left(\frac{\partial^{2} E}{\partial N^{2}}\right)_{v(\vec{r})}
$$

Using a finite difference approximation and Koopmans' theorem, ${ }^{9-12}$ the above expressions can be written as:

$$
\begin{gathered}
\mu=-\frac{1}{2}(I+A) \approx \frac{1}{2}\left(\varepsilon_{L}+\varepsilon_{H}\right)=\chi_{K} \\
\eta=(I-A) \approx\left(\varepsilon_{L}-\varepsilon_{H}\right)=\eta_{K}
\end{gathered}
$$

where $\varepsilon_{\mathrm{H}}$ and $\varepsilon_{\mathrm{L}}$ are the energies of the highest occupied and the lowest unoccupied molecular orbitals, HOMO and LUMO, respectively.

The electrophilicity index $\omega$ has been defined as:

$$
\omega=\frac{\mu^{2}}{2 \eta}=\frac{(I+A)^{2}}{4(I-A)} \approx \frac{\left(\varepsilon_{L}+\varepsilon_{H}\right)^{2}}{4\left(\varepsilon_{L}-\varepsilon_{H}\right)}=\omega_{K}
$$

The electrodonating $\left(\omega^{-}\right)$and electroaccepting $\left(\omega^{+}\right)$powers have been defined as: ${ }^{24}$

$$
\begin{aligned}
& \omega^{-}=\frac{(3 I+A)^{2}}{16(I-A)} \approx \frac{\left(3 \varepsilon_{H}+\varepsilon_{L}\right)^{2}}{16 \eta_{K}}=\omega_{K}^{-} \\
& \omega^{+}=\frac{(I+3 A)^{2}}{16(I-A)} \approx \frac{\left(\varepsilon_{H}+3 \varepsilon_{L}\right)^{2}}{16 \eta_{K}}=\omega_{K}^{+}
\end{aligned}
$$

It follows that a larger $\omega^{+}$value corresponds to a better capability of accepting charge, whereas a smaller value of $\omega^{-}$value of a system makes it a better electron donor. In order to compare $\omega^{+}$with $-\omega^{-}$, the following definition of net electrophilicity has been proposed: ${ }^{25}$

$\Delta \omega^{ \pm}=\omega^{+}-\left(-\omega^{-}\right)=\omega^{+}+\omega^{-} \approx \omega_{K}^{+}-\left(-\omega_{K}^{-}\right)=\omega_{K}^{+}+\omega_{K}^{-}+\Delta \omega_{K}^{ \pm}$

that is, the electroaccepting power relative to the electrodonating power.

\section{SETTINGS AND COMPUTATIONAL METHODS}

All computational studies were performed with the Gaussian $09^{26}$ series of programs with density functional methods as implemented in the computational package. The equilibrium geometries of the molecules were determined by means of the gradient technique. The force constants and vibrational frequencies were determined by computing analytical frequencies on the stationary points obtained after the optimization to check if there were true minima. The basis set used in this work was Def2SVP for geometry optimization and frequencies while Def2TZVP was considered for the calculation of the electronic properties..$^{27,28}$

For the calculation of the molecular structure and properties of the studied systems, we have chosen several density functionals from the Minnesota density functionals family, which consistently provide satisfactory results for several structural and thermodynamic properties: ${ }^{22} \mathrm{M} 11$, which is a range-separated hybrid meta-GGA density functional, ${ }^{29} \mathrm{M} 11 \mathrm{~L}$, which is a dual-range local meta-GGA approximation, ${ }^{30} \mathrm{MN} 12 \mathrm{~L}$, which is a nonseparable local meta-NGA density functional, ${ }^{31} \mathrm{MN} 12 \mathrm{SX}$, which is a range-separated hybrid nonseparable meta-NGA density functional, ${ }^{32} \mathrm{~N} 12$, which is a nonseparable gradient approximation, ${ }^{33} \mathrm{~N} 12 \mathrm{SX}$, which is a range-separated hybrid nonseparable gradient approximation, ${ }^{32}$ SOGGA11, which is a GGA density functional ${ }^{34}$ and SOGGA11X, which is a hybrid GGA density functional. ${ }^{35}$ In these functionals, GGA stands for generalized gradient approximation (in which the density functional depends on the up and down spin densities and their reduced gradient) and NGA stands for nonseparable gradient approximation (in which the density functional depends on the up/down spin densities and their reduced gradient, and also adopts a nonseparable form). All the calculations were performed in the presence of toluene as a solvent, by doing IEF-PCM computations according to the SMD solvation model. ${ }^{36}$ 


\section{RESULTS AND DISCUSSION}

The molecular structures of MgOEP was pre-optimized by starting with the readily available MOL structure, and finding the most stable conformer by means of the Avogadro 1.2.0 program $^{37}$ through a random sampling with molecular mechanics techniques and a consideration of all the torsional angles. The structure of the resulting conformer was then reoptimized with the M11, M11L, MN12L, MN2SX, N12, N12SX, SOGGA11 and SOGGA11X density functionals in connection with the Def2SVP basis set and the SMD solvation model, using toluene as a solvent.

The HOMO and LUMO orbital energies (in eV), global electronegativity $\chi$, total hardness $\eta$, global electrophilicity $\omega$, electrodonating power $\left(\omega^{-}\right)$, electroaccepting power $\left(\omega^{+}\right)$, and net electrophilicity $\Delta \omega^{ \pm}$of the MgOEP molecule calculated with the M11, M11L, MN12L, MN12SX, N12, N12SX, SOGGA11, and SOGGA11X density functionals and the Def2TZVP basis set using toluene as solvent simulated with the SMD parametrization of the IEF-PCM model are presented in Table 1, in which results are shown assuming the validity of the Koopmans' theorem.

The ionization potentials I and electron affinities A (in eV), global electronegativity $\chi$, total hardness $\eta$, global electrophilicity $\omega$, electrodonating power $\left(\omega^{-}\right)$, electroaccepting power $\left(\omega^{+}\right)$, and net electrophilicity $\Delta \omega^{ \pm}$of the MgOEP molecule calculated with the M11, M11L, MN12L, MN12SX, N12, N12SX, SOGGA11, and SOGGA11X density functionals and the Def2TZVP basis set using toluene as as solvent simulated with the SMD parametrization of the IEF-PCM model are presented in Table 2, in which results are derived from the calculated vertical I and A.
Inspired from previous works on this subject, ${ }^{13,21}$ and with the object of analyzing our results in order to verify the fulfillment of the "Koopmans' theorem in DFT", we have designed several descriptors that relate the results obtained through the HOMO and LUMO calculations with those obtained by means of the vertical I and A with a $\triangle \mathrm{SCF}$ procedure. However, it must be stressed that it is not our intention to perform a gap-fitting by minimizing a descriptor by choosing optimal range-separation parameter $\gamma$, but to check if the density functionals considered in this study, in which, some of the contain a fixed range-separation parameter $\gamma$, obbey the "Koopmans' theorem in DFT". As a matter fact, there is no range-separation parameter $\gamma$ in our designed descriptors. Moreover, we have considered A as minus the energy of the LUMO of the neutral system instead of considering A as minus the energy of the HOMO of the $\mathrm{N}+1$ electron system, as it was in the mentioned works. ${ }^{13,21}$

The first three descriptors are related to the simplest fulfillment of the Koopmans' theorem by relating $\varepsilon_{\mathrm{H}}$ with $-\mathrm{I}, \varepsilon_{\mathrm{L}}$ with $-\mathrm{A}$, and the behavior of them in the description of the HOMO-LUMO gap:

$$
\begin{gathered}
J_{I}=\left|\varepsilon_{H}+E_{g s}(N-1)-E_{g s}(N)\right| \\
J_{A}=\left|\varepsilon_{L}+E_{g s}(N)-E_{g s}(N+1)\right| \\
J_{H L}=\sqrt{J_{I}^{2}+J_{A}^{2}}
\end{gathered}
$$

Next, we consider four other descriptors that analyze how well the studied density functionals are useful for the prediction of the electronegativity $\chi$, the global hardness $\eta$ and the global electrophilicity $\omega$, and for a combination of these Conceptual DFT descriptors, just

Table 1. HOMO and LUMO orbital energies (in eV), global electronegativity $\chi$, total chemical hardness $\eta$, global electrophilicity $\omega$, electrodonating power $\left(\omega^{-}\right)$, electroaccepting power $\left(\omega^{+}\right)$, and net electrophilicity $\Delta \omega^{ \pm}$of magnesium octaethylporphyrin (MgOEP) calculated with the M11, M11L, MN12L, MN12SX, N12, N12SX, SOGGA11 and SOGGA11X density functionals and the Def2TZVP basis set using toluene as solvent simulated with the SMD parametrization of the IEF-PCM model

\begin{tabular}{lllllllll}
\hline Property & M11 & M11L & MN12L & MN12SX & N12 & N12SX & SOGGA11 & SOGGA11X \\
\hline HOMO & -5.389 & -3.254 & -2.857 & -3.253 & -2.807 & -3.120 & -3.096 & -3.983 \\
\hline LUMO & -0.267 & -2.578 & -2.107 & -2.125 & -2.139 & -1.988 & -2.473 & -1.358 \\
\hline$\chi_{\mathrm{K}}$ & 2.828 & 2.916 & 2.482 & 2.689 & 2.473 & 2.554 & 2.784 & 2.671 \\
\hline$\eta_{\mathrm{K}}$ & 5.122 & 0.676 & 0.750 & 1.128 & 1.668 & 0.132 & 0.623 & 2.625 \\
\hline$\omega_{\mathrm{K}}$ & 0.781 & 6.290 & 4.106 & 3.204 & 4.579 & 2.882 & 6.226 & 1.358 \\
\hline$\omega_{\mathrm{K}}{ }^{+}$ & 3.295 & 14.080 & 9.500 & 7.824 & 10.436 & 7.112 & 13.882 & 4.216 \\
\hline$\omega_{\mathrm{K}^{+}}$ & 0.467 & 11.164 & 7.018 & 5.135 & 7.963 & 4.557 & 11.098 & 1.545 \\
\hline$\Delta \omega_{\mathrm{K}}{ }^{+}$ & 3.762 & 25.244 & 15.518 & 12.959 & 18.399 & 11.669 & 24.981 & 5.761 \\
\hline
\end{tabular}

Table 2. Ionization potentials I and electron affinities (in $\mathrm{eV}$ ), global electronegativity $\chi$, total chemical hardness $\eta$, global electrophilicity $\omega$, electrodonating power $\left(\omega^{-}\right)$, electroaccepting power $\left(\omega^{+}\right)$, and net electrophilicity $\Delta \omega^{ \pm}$of magnesium octaethylporphyrin (MgOEP) calculated with the M11, M11L, MN12L, MN12SX, N12, N12SX, SOGGA11 and SOGGA11X density functionals and the Def2TZVP basis set using toluene as solvent simulated with the SMD parametrization of the IEF-PCM model

\begin{tabular}{lcccccccc}
\hline Property & M11 & M11L & MN12L & MN12SX & N12 & N12SX & SOGGA11 & SOGGA11X \\
\hline $\mathbf{I}$ & 3.596 & 3.254 & 2.953 & 3.239 & 2.836 & 3.071 & 3.335 & 3.306 \\
$\mathbf{A}$ & 2.142 & 2.502 & 1.984 & 2.171 & 2.053 & 2.059 & 2.289 & 2.086 \\
$\chi$ & 2.869 & 2.878 & 2.468 & 2.705 & 2.445 & 2.565 & 2.812 & 2.696 \\
$\eta$ & 1.454 & 0.752 & 0.969 & 1.068 & 0.783 & 1.012 & 1.047 & 1.220 \\
$\omega$ & 2.830 & 5.507 & 3.142 & 3.426 & 3.814 & 3.251 & 3.778 & 2.980 \\
$\omega^{-}$ & 7.186 & 12.500 & 7.579 & 8.271 & 8.898 & 7.847 & 9.027 & 7.384 \\
$\omega^{+}$ & 4.317 & 9.622 & 5.111 & 5.566 & 6.454 & 5.283 & 6.215 & 4.688 \\
$\Delta \omega_{\mathbf{K}}{ }^{+}$ & 11.503 & 22.122 & 12.691 & 13.837 & 15.352 & 13.130 & 15.242 & 12.071 \\
\hline
\end{tabular}


considering the energies of the HOMO and LUMO or the vertical I and A:

$$
\begin{gathered}
J_{\chi}=\left|\chi-\chi_{K}\right| \\
J_{\eta}=\left|\eta-\eta_{K}\right| \\
J_{\omega}=\left|\omega-\omega_{K}\right| \\
J_{D 1}=\sqrt{J_{\chi}^{2}+J_{\eta}^{2}+J_{\omega}^{2}}
\end{gathered}
$$

where D1 stands for the first group of Conceptual DFT descriptors.

Finally, we designed other four descriptors to verify the goodness of the studied density functionals for the prediction of the electroaccepting power $\omega^{+}$, the electrodonating power $\omega^{-}$, the net electrophilicity $\Delta \omega^{ \pm}$, and for a combination of these Conceptual DFT descriptors, just considering the energies of the HOMO and LUMO or the vertical I and A:

$$
\begin{gathered}
J_{\omega^{+}}=\left|\omega^{+}-\omega_{K}^{+}\right| \\
J_{\omega^{-}}=\left|\omega^{-}-\omega_{K}^{-}\right| \\
J_{\Delta \omega^{ \pm}}=\left|\Delta \omega^{ \pm}-\Delta \omega_{K}^{ \pm}\right| \\
J_{D 2}=\sqrt{J_{\omega^{-}}^{2}+J_{\omega^{+}}^{2}+J_{\Delta \omega^{ \pm}}^{2}}
\end{gathered}
$$

where D2 stands for the first group of Conceptual DFT descriptors.

The results of the calculations of $\mathrm{J}_{\mathrm{I}}, \mathrm{J}_{\mathrm{A}}, \mathrm{J}_{\mathrm{HL}}, \mathrm{J}_{\chi}, \mathrm{J}_{\eta}, \mathrm{J}_{\omega}, \mathrm{J}_{\mathrm{D} 1}, \mathrm{~J}_{\omega^{+}}$, $\mathrm{J}_{\omega^{-}}, \mathrm{J}_{\Delta \omega^{ \pm}}$and JD2 for the MgOEP molecule are displayed in Table 3.

As can be seen from Tables 1 and 2, and the results presented in Table 3, the "Koopmans' theorem in DFT" holds with great accuracy for the MN12SX and N12SX density functionals, which are a range-separated hybrid meta-NGA and a range-separated hybrid NGA density functionals, respectively. Indeed, the values of $J_{I}, J_{A}$ and $\mathrm{J}_{\mathrm{HL}}$ are not exactly zero. However, their values can be favorably compared with the results presented for these quantities in the work of Lima et al. ${ }^{21}$ where the minima have been obtained by choosing a parameter that enforces that behavior. It is interesting to see that the same density functionals also fulfill the "Koopmans' theorem in DFT" procedure for the other descriptors, namely $\mathrm{J}_{\chi}, \mathrm{J}_{\eta}, \mathrm{J}_{\omega}$, and $\mathrm{J}_{\mathrm{D} 1}$, as well as for $\mathrm{J}_{\omega^{-}}, \mathrm{J}_{\omega^{+}}, \mathrm{J}_{\Delta \omega^{ \pm}}$, and $\mathrm{J}_{\mathrm{D} 2}$. These results are very important, because they show that it is not enough to rely only in $\mathrm{J}_{\mathrm{I}}$,
$\mathrm{J}_{\mathrm{A}}$ and $\mathrm{J}_{\mathrm{HL}}$. For example, if we consider only $\mathrm{J}_{\chi}$, for all the studied density functionals, the values are very close to zero. As for the other descriptors, only the MN12SX and N12SX density functionals show this behavior. That means that the results for $\mathrm{J}_{\chi}$ are due to a fortuitous cancellation of errors.

The usual GGA (SOGGA11) and hybrid-GGA (SOGGA11X) presented greater deviations in the results and were therefore less adequate for the fulfillment of the "Koopmans' theorem in DFT" procedure, and the same conclusion is valid for the local functionals M11L, MN12L and N12, as well as for the M11 density functional.

An important fact is that although the range-separated hybrid NGA and range-separated hybrid meta-NGA density functionals can be useful for the calculation of the Conceptual DFT descriptors, it is not the same for the range-separated hybrid GGA (M11) density functional. An inspection of Tables 1 and 2 shows that this is due to the fact that this functional describes inadequately the energy of the LUMO.

\section{CONCLUSIONS}

From the whole of the results presented in this contribution it has been clearly demonstrated that the chemical reactivity of the MgOEP molecule can be predicted by using DFT-based reactivity descriptors such as the electronegativity, global hardness, global electrophilicity, electrodonating and electroaccepting powers, and net electrophilicity.

The Minnesota family of density functionals (M11, M11L, MN12L, MN12SX, N12, N12SX, SOGGA11 and SOGGA11X) have been tested for the fulfillment of the "Koopmans' theorem in DFT" by comparison of the HOMO- and LUMO- derived values with those obtained through a $\triangle \mathrm{SCF}$ procedure. It has been shown that the range-separated hybrid meta-NGA density functional (MN12SX) and the range-separated hybrid NGA density functional (N12SX) are the best for the accomplishment of this objective. One of the possible explanations for this behavior lies on the fact they are screened-exchange hybrid density functionals. The approach considered in these density functionals uses a finite amount of HF exchange at short-range, but none in the long-range limit, in order to cut the computational cost of non-local exchange integrals for

\begin{tabular}{|c|c|c|c|c|c|c|c|c|}
\hline Descriptor & M11 & M11L & MN12L & MN12SX & N12 & N12SX & SOGGA11 & SOGGA11X \\
\hline $\mathbf{J}_{\mathbf{I}}$ & 1.792 & 0.000 & 0.096 & 0.014 & 0.029 & 0.050 & 0.240 & 0.678 \\
\hline $\mathbf{J}_{\mathbf{A}}$ & 1.875 & 0.076 & 0.123 & 0.046 & 0.087 & 0.071 & 0.184 & 0.728 \\
\hline $\mathbf{J}_{\mathrm{HL}}$ & 2.594 & 0.076 & 0.156 & 0.048 & 0.091 & 0.086 & 0.302 & 0.995 \\
\hline $\mathbf{J}_{\chi}$ & 0.041 & 0.038 & 0.013 & 0.016 & 0.029 & 0.011 & 0.028 & 0.025 \\
\hline $\mathbf{J}_{\eta}$ & 3.668 & 0.076 & 0.220 & 0.060 & 0.115 & 0.120 & 0.424 & 1.406 \\
\hline $\mathbf{J}_{\omega}$ & 2.050 & 0.783 & 0.964 & 0.221 & 0.765 & 0.369 & 2.448 & 1.621 \\
\hline $\mathbf{J}_{\mathbf{D} 1}$ & 4.202 & 0.787 & 0.989 & 0.230 & 0.774 & 0.388 & 2.485 & 2.146 \\
\hline $\mathbf{J}_{\omega^{-}}$ & 3.891 & 1.580 & 1.921 & 0.447 & 1.538 & 0.736 & 4.856 & 3.168 \\
\hline $\mathbf{J}_{\omega^{+}}$ & 3.849 & 1.542 & 1.907 & 0.431 & 1.509 & 0.725 & 4.883 & 3.142 \\
\hline $\mathbf{J}_{\Delta \omega^{ \pm}}$ & 7.740 & 3.122 & 3.828 & 0.878 & 3.047 & 1.461 & 9.739 & 6.310 \\
\hline $\mathbf{J}_{\mathbf{D} 2}$ & 9.480 & 3.824 & 4.688 & 1.076 & 3.731 & 1.789 & 11.928 & 7.728 \\
\hline
\end{tabular}
extended systems. Therefore, they are a good alternative to those density functionals whose behavior have been tuned through a gap-fitting procedure and a good prospect for their usefulness in the description of the chemical reactivity of porphyrin molecular systems of larger sizes.

Table 3. Descriptors $\mathrm{J}_{\mathrm{I}}, \mathrm{J}_{\mathrm{A}}, \mathrm{J}_{\mathrm{HL}}, \mathrm{J}_{\chi}, \mathrm{J}_{\eta}, \mathrm{J}_{\omega}, \mathrm{J}_{\mathrm{D} 1}, \mathrm{~J}_{\omega^{+}}, \mathrm{J}_{\omega^{-}}, \mathrm{J}_{\Delta \omega^{ \pm}}$and JD2 for the magnesium octaethylporphyrin (MgOEP) molecule calculated from the results of Tables 1 and 2 


\section{ACKNOWLEDGEMENTS}

This work has been partially supported by CIMAV, SC and Consejo Nacional de Ciencia y Tecnología (CONACYT, Mexico) through Grant 219566/2014 for Basic Science Research and Grant 265217/2016 for a Foreign Sabbatical Leave. Daniel GlossmanMitnik conducted this work while a Sabbatical Fellow at the University of the Balearic Islands from which support is gratefully acknowledged. This work was cofunded by the Ministerio de Economía y Competitividad (MINECO) and the European Fund for Regional Development (FEDER) (CTQ2014-55835-R).

\section{REFERENCES}

1. Johnson, B. J.; Taitt, C. R.; Gleaves, A; North, S. H.; Malanoski, A. P.; Leska, I. A.; Archibong, E.; Monk, S. M.; Sensing and Bio-Sensing Research 2016, 8, 1 .

2. Moin, S. T.; Hofer, T. S.; Mol. BioSyst. 2014, 10, 117.

3. Parr, R.; Yang, W.; Density-Functional Theory of Atoms and Molecules, Oxford University Press: New York, 1989.

4. Geerlings, P.; De Proft, F.; Langenaeker, W.; Chem. Rev. 2003, 103, 1793.

5. Toro-Labbé, A. In Theoretical Aspects of Chemical Reactivity; ToroLabbé, A., ed.; Elsevier Science: Amsterdam, 2007.

6. Chattaraj, P. K. In Chemical Reactivity Theory - A Density Functional View; Chattaraj, P. K., ed.; CRC Press - Taylor \& Francis Group: Boca Raton, 2009.

7. Huzinaga, S.; Andzelm, J.; Klobukowski, M.; Radzio-Audzelm, E.; Sakai, Y.; Tatewaki, H.; Gaussian Basis Sets for Molecular Calculations, Elsevier: Amsterdam, 1984.

8. Easton, R.; Giesen, D.; Welch, A.; Cramer, D.; Truhlar, D.; Theor. Chem. Acc. 1996, 93, 281.

9. Lewars, E.; Computational Chemistry - Introduction to the Theory and Applications of Molecular and Quantum Mechanics, Kluwer Academic Publishers: Dordrecht, 2003.

10. Young, D.; Computational Chemistry - A Practical Guide for Applying Techniques to Real-World Problems, John Wiley \& Sons: New York, 2001.

11. Jensen, F.; Introduction to Computational Chemistry, $2^{\text {nd }}$ Edition, John Wiley \& Sons: Chichester, 2007.

12. Cramer, C.; Essentials of Computational Chemistry - Theories and Models, $2^{\text {nd }}$ Edition, John Wiley \& Sons: Chichester, 2004.

13. Kronik, L.; Stein, T.; Refaely-Abramson, S.; Baer, R.; J. Chem. Theory Comput. 2012, 8, 1515.

14. Perdew, J.; Parr, R.; Levy, M.; Balduz, J. L. J.; Phys. Rev. Lett. 1982, 49, 1691.
15. Almbladh, C.-O.; von Barth, U.; Phys. Rev. B 1985, 31, 3231.

16. Perdew, J.; Burke, K.; Ersernhof, M.; Phys. Rev. Lett. 1997, 78, 1396.

17. Levy, M.; Perdew, J. P.; Sahni, V.; Phys. Rev. A 1984, 30, 2745.

18. Savin, A. In Recent Advances in Density Functional Methods; Chong, D. P., ed.; World Scientific: Singapore, 1995.

19. Leininger, T.; Stoll, H.; Werner, H.-J.; Savin, A.; Chem. Phys. Lett. 1997, 275,151 .

20. Savin, A.; Flad, H.-J.; Int. J. Quantum Chem. 1995, 56, 327.

21. Lima, I. T.; Prado, A. d. S.; Martins, J. B. L.; de Oliveira Neto, P. H.; Ceschin, A. M., J. Phys. Chem. A 2016, 120, 4944.

22. Peverati, R.; Truhlar, D. G.; Philos. Trans. R. Soc., A 2014, 372, 20120476.

23. Parr, R.; Yang, W.; J. Am. Chem. Soc. 1984, 106, 4049.

24. Gázquez, J.; Cedillo, A.; Vela, A.; J. Phys. Chem. A 2007, 11, 1966.

25. Chattaraj, P.; Chakraborty, A.; Giri, S.; J. Phys. Chem. A 2009, 113, 10068.

26. Frisch, M. J.; Trucks, G. W.; Schlegel, H. B.; Scuseria, G. E.; Robb, M. A.; Cheeseman, J. R.; Scalmani, G.; Barone, V.; Petersson, G. A.; Nakatsuji, H.; Li, X.; Caricato, M.; Marenich, A. V.; Bloino, J.; Janesko, B. G.; Gomperts, R.; Mennucci, B.; Hratchian, H. P.; Ortiz, J. V.; Izmaylov, A. F.; Sonnenberg, J. L.; Williams-Young, D.; Ding, F.; Lipparini, F.; Egidi, F.; Goings, J.; Peng, B.; Petrone, A.; Henderson, T.; Ranasinghe, D.; Zakrzewski, V. G.; Gao, J.; Rega, N.; Zheng, G.; Liang, W.; Hada, M.; Ehara, M.; Toyota, K.; Fukuda, R.; Hasegawa, J.; Ishida, M.; Nakajima, T.; Honda, Y.; Kitao, O.; Nakai, H.; Vreven, T.; Throssell, K.; Montgomery, J. A., Jr.; Peralta, J. E.; Ogliaro, F.; Bearpark, M. J.; Heyd, J. J.; Brothers, E. N.; Kudin, K. N.; Staroverov, V. N.; Keith, T. A.; Kobayashi, R.; Normand, J.; Raghavachari, K.; Rendell, A. P.; Burant, J. C.; Iyengar, S. S.; Tomasi, J.; Cossi, M.; Millam, J. M.; Klene, M.; Adamo, C.; Cammi, R.; Ochterski, J. W.; Martin, R. L.; Morokuma, K.; Farkas, O.; Foresman, J. B.; Fox, D. J.; Gaussian 09 Revision D.01, Gaussian Inc., Wallingford CT, 2009.

27. Weigend, F.; Ahlrichs, R.; Phys. Chem. Chem. Phys. 2005, 7, 3297.

28. Weigend, F.; Phys. Chem. Chem. Phys. 2006, 8, 1057.

29. Peverati, R.; Truhlar, D. G.; J. Phys. Chem. Lett. 2011, 2, 2810.

30. Peverati, R; Truhlar, D. G.; J. Phys. Chem. Lett. 2012, 3, 117.

31. Peverati, R.; Truhlar, D. G.; Phys. Chem. Chem. Phys. 2012, 14, 13171.

32. Peverati, R.; Truhlar, D. G.; Phys. Chem. Chem. Phys. 2012, 14, 16187.

33. Peverati, R.; Truhlar, D. G.; J. Chem. Theory Comput. 2012, 8, 2310.

34. Peverati, R.; Zhao, Y.; Truhlar, D. G.; J. Phys. Chem. Lett. 2011, $2,1991$.

35. Peverati, R.; Truhlar, D. G.; J. Chem. Phys. 2011, 135, 191102.

36. Marenich, A.; Cramer, C.; Truhlar, D.; J. Phys. Chem. B 2009, 113, 6378.

37. Hanweel, M.; Curtis, D. E., Lonie, D.; Vandermeersch, T.; Zurek, E.; Hutchison, G.; J. Cheminf. 2012, 4, 17. 